\title{
Integrative analysis of cancer driver genes in prostate adenocarcinoma
}

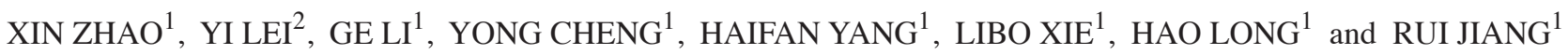 \\ Departments of ${ }^{1}$ Urology and ${ }^{2}$ Endocrinology, The Affiliated Hospital of \\ Southwest Medical University, Luzhou, Sichuan 646000, P.R. China
}

Received June 12, 2018; Accepted January 4, 2019

DOI: $10.3892 / \mathrm{mmr} .2019 .9902$

\begin{abstract}
Large-scale genomics studies have identified recurrently mutated genes in the ETS gene family, including fusions and copy number variations (CNVs), which are involved in the development of prostate adenocarcinoma (PRAD). However, the aetiology of PRAD remains to be fully elucidated. In the present study, 333 driver genes were identified using four computational tools: OncodriveFM, OncodriveCLUST, iCAGES and DrGaP. In addition, 32 driver pathways were identified using DrGaP. SPOP, TP53, SPTA1, AHNAK, HMCN1, ATM, FOXA1, CSMD3, LRPIB and FREM2 were the 10 most recurrently mutated genes in PRAD. ITGAL, TAGAP, SIGLEC10, RAC2 and ITGA4 were the five hub genes in the yellow module that were associated with the number of positive lymph nodes. Hierarchical clustering analysis of the 20 driver genes with the most frequent CNVs revealed three clusters of patients with PRAD. Cluster 3 tumours exhibited significantly higher numbers of positive lymph nodes, higher Gleason scores, more advanced cancer stages and poorer prognosis than cluster 1 and 2 tumours. A total of 48 genes were significantly associated with the number of positive lymph nodes, Gleason scores and pathologic stage in patients with PRAD. The identified set of cancer genes and pathways sheds light on the tumorigenesis of PRAD and creates avenues for the development of prognostic biomarkers and driver gene-targeted therapies in PRAD.
\end{abstract}

Correspondence to: Dr Rui Jiang, Department of Urology, The Affiliated Hospital of Southwest Medical University, 25 Taiping Road, Luzhou, Sichuan 646000, P.R. China

E-mail: jiangruilyfy@163.com

Abbreviations: PRAD, prostate adenocarcinoma; TCGA, The Cancer Genome Atlas; TSS, transcription start site; iCAGES, integrated CAncer GEnome Score; GO, Gene Ontology; WGCNA, weighted correlation network analysis; KEGG, Kyoto Encyclopaedia of Genes and Genomes; STRING, Search Tool for the Retrieval of Interacting Genes/Proteins; CNV, copy number variation; FDR, false discovery rate

Key words: prostate adenocarcinoma, driver gene, driver pathway

\section{Introduction}

Prostate cancer is the second most common type of cancer in men worldwide and accounts for an annual mortality rate of $>250,000$ (1). The most common subtype of prostate cancer is prostate adenocarcinoma (PRAD), which expresses the androgen receptor, while other categories of prostate cancer, including mucinous carcinoma, signet ring cell carcinoma, neuroendocrine prostate cancer, adenosquamous and squamous cell carcinoma, are relatively rare $(2,3)$. Prostate tumours are biologically heterogeneous diseases, with some patients succumbing to mortality from metastases within 2-3 years and others surviving for 10-20 years following diagnosis with localised disease.

Over the last 10 years, characterization of the prostate cancer transcriptome and genome has revealed recurrent somatic mutations, chromosomal rearrangements, and copy number gains and losses, enhancing understanding of PRAD tumorigenesis. Barbieri et al performed exome-sequencing on 112 cases of prostate adenocarcinoma and found that the most frequently mutated genes in primary prostate cancer were SPOP, TP53, FOXA1 and PTEN. Prostate cancer with mutant SPOP lacks ETS family gene rearrangements and exhibits a distinct pattern of genomic alterations, characterised by the enrichment of both 5q21 and 6q21 deletions (4). A comprehensive molecular analysis of 333 samples of primary prostate carcinoma revealed that $53 \%$ of tumours had ETS family gene fusions, and TMPRSS2 was the most frequent fusion partner among all ETS fusions (5). PRAD can be classified into seven distinct subtypes, defined by ERG fusions, ETV1/ETV4/FLI1 fusions or overexpression, or by SPOP, FOXA1 and IDH1 mutations (6). Taylor et al found that the nuclear receptor coactivator NCOA2 functions as an oncogene in $11 \%$ of PRAD tumours. The combined loss of $13 \mathrm{q}$ and $18 \mathrm{q}$, focal amplification of two distinct $5 p$ regions (5p13 or $5 \mathrm{p} 15)$, and focal deletion of $5 \mathrm{q} 21.1$ are each significantly associated with negative clinical outcome (5).

Previous studies have mainly focused on genes that are recurrently mutated in PRAD samples; however, several driver genes may occur at a low frequency. For example, certain cancer drivers are mutated in a small fraction (e.g., <1\%) of tumours (7). Therefore, investigations may overlook potential drivers that are mutated at a low frequency in PRAD, and no investigations have been conducted on the classification 
of patients with PRAD using low mutation frequency genes. In the present study, integrated analyses were performed on 332 PRAD samples using diverse omics data types from The Cancer Genome Atlas (TCGA) database (6). The results revealed a list of novel driver genes and driver pathways, and revealed three distinct subgroups of patients with PRAD, providing a better understanding of this disease and suggesting potential therapeutic targets in PRAD.

\section{Materials and methods}

Classification of somatic mutations in PRAD. A total of 12,348 somatic mutations of 332 pairs of PRAD tumour/normal samples were accessed from the Broad Institute (http://gdac. broadinstitute.org/) (6). The Ensembl Variant Effect Predictor (https://asia.ensembl.org/info/docs/tools/vep/index.html) was used to assess the functional impact of somatic mutations (8) and the mutations were then divided into nine groups based on their functional impact, including frame shift indels, in-frame indels, missense mutation, nonsense mutation, nonstop mutation, RNA, silent, splice site and translation start site (TSS). RNA indicated mutation in the 5'untranslated region (UTR) or 3'UTR that may be functional but likely via effects on the RNA level.

Prediction of driver genes and pathways in PRAD. OncodriveCLUST groups protein positions with a number of mutations expected by chance to form mutation clusters. Each cluster is scored with a value proportional to the percentage of gene mutations that are enclosed within that cluster and inversely related to its length. A gene clustering score is the sum of the scores of all clusters (if any) found in that gene. OncodriveCLUST 0.4.1 (https://www.intogen.org/analysis/) constructs the background model by assessing coding-silent mutations and identifies genes with a significant bias towards mutation clustering within the protein sequence (9). OncodriveFM 0.0.1 (https://www.intogen.org/analysis/) (10) first uses three tools, SIFT (11), PolyPhen2 (12) and MutationAssessor (13), to compute the functional impact score of a somatic mutation. These functional scores are then transformed into a uniform score that measures the damaging impact of somatic mutations using transFIC (14). OncodriveFM computes bias towards the accumulation of variants with high functional impact to identify drivers by comparing the actual functional impact with a null distribution model generated by 1,000,000 permutations. Genes with $\mathrm{Q}<0.05$ are considered driver genes by OncodriveCLUST and OncodriveFM.

The integrated CAncer GEnome Score (iCAGES, http://wglab.org/software/11-icages) developed by Wang Genomics Lab is a novel statistical framework that infers driver variants by integrating contributions from coding, noncoding and structural variants, identifying driver genes by combining genomic information and prior biological knowledge to generate prioritised drug treatments (15). iCAGES consists of three consecutive layers. The first layer prioritises personalised cancer driver coding, noncoding and structural variations. The second layer associates these mutations to genes using a statistical model with prior biological knowledge of cancer driver genes for specific subtypes of cancer. The third layer generates a list of drugs targeting the repertoire of these potential driver genes. Genes with iCAGESGeneScores $>0.5$ are determined as drivers by iCAGES.

DrGaP 0.1.0 (http://code.google.com/p/drgap/) developed by Dr Lu's lab at the Medical College of Wisconsin (16) comprises statistical methods and several auxiliary bioinformatics tools to detect driver genes and driver signalling pathways in cancer genome-sequencing. The statistical methods use a Poisson process to model the random nature of somatic mutations and a Bayesian model to estimate background mutation rates. A likelihood ratio test is conducted to determine the significance of driver genes and pathways. DrGaP integrates biological knowledge of the mutational process in tumours, including the length of protein-coding regions, transcript isoforms, variation in mutation types, differences in background mutation rates, redundancy of the genetic code and multiple mutations in one gene. Genes or pathways with P-values $<0.05$ are regarded as driver genes or pathways by DrGaP.

Gene Ontology (GO) and Kyoto Encyclopaedia of Genes and Genomes (KEGG) pathway enrichment analyses in PRAD. The GO terms and KEGG pathway enrichment analyses were performed with GO (http://geneontology.org) (17) and Search Tool for the Retrieval of Interacting Genes/Proteins (STRING 10.5; http://string.embl.de) (18), respectively, to characterise the functional enrichment of all driver genes. The driver genes were considered to be significantly enriched in GO terms or KEGG pathways using the cut-offs of $\mathrm{P}<0.05$ or false discovery rate $(\mathrm{FDR})<0.05$.

Coexpression network analyses in PRAD. Coexpression networks were constructedusing theR package of weighted correlation network analysis (WGCNA; https://horvath.genetics. ucla.edu/html/CoexpressionNetwork/Rpackages/WGCNA/) with RNA-seq expression data expressed as normalised read counts of driver genes in 497 patients with PRAD (19). All parameters were set to default values, with the exception of softpower (7). The minimum number of genes was set as 10 to ensure reliability of the results. Genes with a high intramodular connectivity are considered intramodular hub genes. The clinical traits of 497 patients with PRAD were obtained from the TCGA database. Module-trait associations were estimated using the correlation between the module eigengene and clinical traits, which enables easy identification of modules showing high correlation with clinical features.

Copy number variation (CNV) analyses in PRAD. Focal CNVs and genes with significant gains and losses in 492 PRAD samples were detected using the GISTIC algorithm (20) and accessed at the Broad Institute (6). Unsupervised hierarchical clustering of 20 driver genes with CNVs at the highest frequency was conducted using the function heatmap. 2 of the R package of gplots (21). Patient age, number of positive lymph nodes, cancer stage, and Gleason scores were compared among patients in the three clusters using the Wilcoxon sum rank test. Kaplan-Meier curves were plotted using the $\mathrm{R}$ package of survival (22), and survival rates were compared among patients in the three clusters using the log-rank test. $\mathrm{P}<0.05$ was predefined as statistically significant. 
A

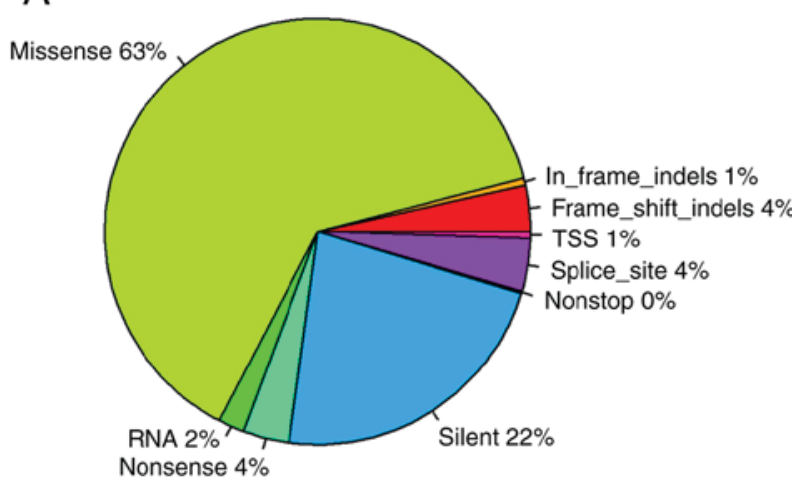

B

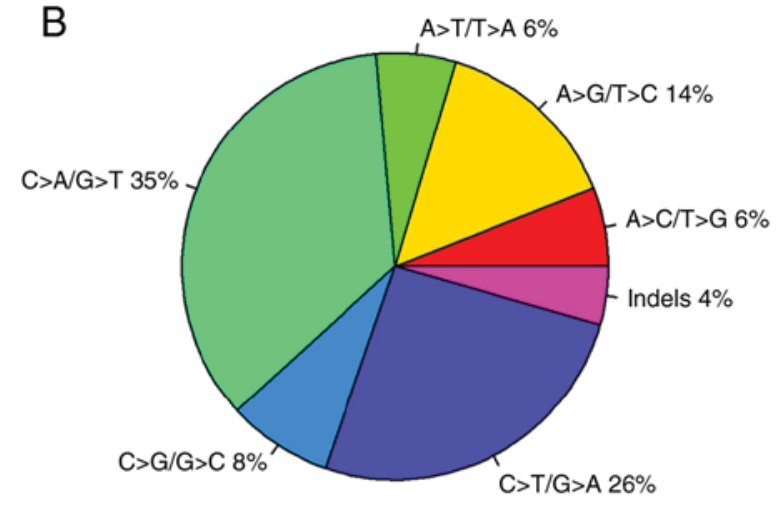

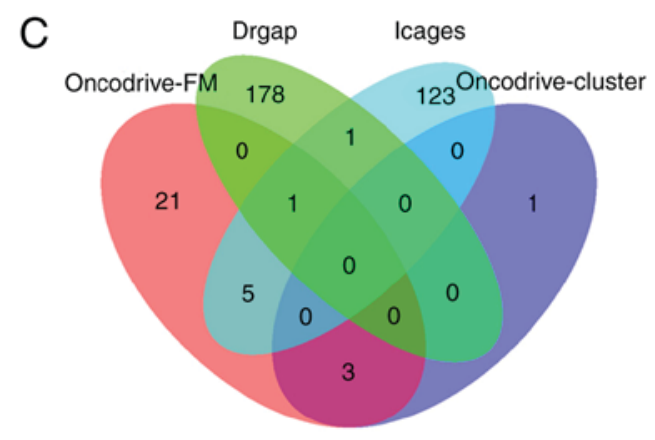

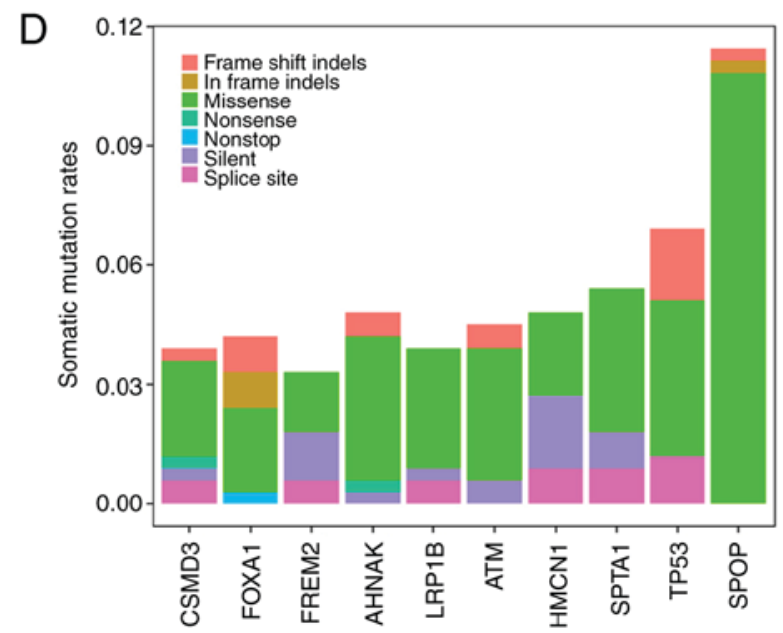

Figure 1. Characterization of somatic mutations and cancer genes in PRAD. (A) Number and proportion of mutation classes with different functional impacts in PRAD. (B) Somatic mutation signatures in PRAD. (C) Overlap of driver genes detected by OncodriveFM, OncodriveCLUST, iCAGES and DrGaP in patients with PRAD. (D) Mutation rates of the 10 most frequently mutated driver genes in 332 PRAD samples. PRAD, prostate adenocarcinoma; iCAGES, integrated CAncer GEnome Score; TSS, transcription start site.

Clinical features analyses in PRAD. The clinical features of 497 patients with PRAD were downloaded from the Broad Institute and included patient outcome, number of positive lymph nodes, Gleason scores and pathologic stages. For survival analysis, a log-rank test in univariate Cox regression analysis with a proportional hazards model (23) was used to estimate the P-values, comparing quantile intervals using the 'coxph' function in R (22). Driver genes were considered to be significantly associated with survival at $\mathrm{P}<0.05$. For the number of positive lymph nodes, Gleason scores and pathologic stages, Spearman's rank correlation was performed between each feature type and gene expression ( $\log 2$ normalised read count) using the 'cor.test' function in R. Driver genes were considered to be significantly associated with positive lymph nodes, Gleason scores, or pathologic stages at $\mathrm{P}<0.05$ and $\mathrm{q}<0.05$.

Validation of randomised selection of patients with PRAD. The datasets used for the somatic mutation, coexpression network, clinical feature and CNV analyses were distinct patient cohorts. To evaluate heterogeneity across the different cohorts, clinical characteristics were compared among the three cohorts of patients with PRAD. Patient age, number of positive lymph nodes and Gleason scores were compared using the Wilcoxon sum rank test. Cancer stages were compared using Pearson's $\chi^{2}$ test. Kaplan-Meier curves were plotted using the R package of survival (22), and survival rates were compared among patients in the three cohorts using the log-rank test. $\mathrm{P}<0.05$ was predefined as statistically significant.

\section{Results}

Somatic mutations, driver genes and pathways in PRAD. In total, 12,348 somatic mutations were detected in 332 PRAD samples, with an average mutation density of 0.29 somatic mutations per megabase per sample, which is lower than that observed in other cancer types, including melanoma and lung cancer (24). The somatic mutations comprised 7,816 missense, 2,779 silent, 496 splice-site, 433 nonsense, 246 RNA, 64 TSS, and 12 nonstop mutations, and 402 insertions or deletions (indels). Of the 402 indels, 334 caused reading frame shifts, and 68 indels were located in open reading frames. Nonsynonymous mutations accounted for $63.3 \%(7,816 / 12,348)$ of the variants in PRAD (Fig. 1A). C $>\mathrm{T} / \mathrm{G}>\mathrm{A}, \mathrm{C}>\mathrm{T} / \mathrm{G}>\mathrm{A}$ and $\mathrm{A}>\mathrm{G} / \mathrm{T}>\mathrm{C}$ were the three predominant transitions, with mutation rates of 35.4 , 25.8 and $14.4 \%$, respectively (Fig. 1B).

Overall, 7,471 genes were mutated in at least one PRAD sample. There were four, 30, 130 and 180 driver genes predicted by OncodriveCLUST, OncodriveFM, iCAGES and DrGaP, respectively (data not shown). Combining the four sets of driver genes, a total of 333 unique driver genes were detected 
by all four tools. SPOP, FOXA1 and MED12 were overlapping genes identified by OncodriveCLUST and OncodriveFM. TP53, BRAF, CTNNB1, BRCA2, SMAD4 and AKT1 were common to OncodriveFM and iCAGES. ERBB3 and TP53 were common driver genes to iCAGES and DrGaP. TP53 was the only driver gene predicted by OncodriveFM, iCAGES and DrGaP (Fig. 1C). Among the 333 driver genes, SPOP, TP53, SPTA1, AHNAK, HMCN1, ATM, FOXA1, CSMD3, LRPIB and FREM2 were the 10 most recurrently mutated genes in PRAD, with mutation rates of $11.45,6.93,5.42,4.82,4.82$, $4.52,4.22,3.92,3.92$ and $3.31 \%$, respectively, across all PRAD samples (Fig. 1D). By contrast, the majority of driver genes were low-frequency mutated genes in PRAD, with an average mutation rate of $0.83 \%$ (data not shown). In addition to the list of driver genes, DrGaP identified 66 driver pathways in PRAD, including the p53 signalling pathway, the wnt signalling pathway, the MAPK signalling pathway, glioma, thyroid cancer, apoptosis, and pathways in cancer (data not shown).

GO terms and KEGG pathway enrichment analyses. The enrichment of GO terms and KEGG pathways was analysed for 333 driver genes using the GO and STRING tools. $\mathrm{GO}$ enrichment analysis indicated that driver genes were significantly overrepresented in 1,563 biological processes (adjusted $\mathrm{P}<0.05$, data not shown). The main GO biological processes exhibited a wide spectrum of functional processes, including cell proliferation, regulation of mitotic cell cycle, cell differentiation, regulation of apoptotic processes, regulation of cell death, and regulation of cellular metabolic processes. STRING also revealed $135 \mathrm{KEGG}$ pathways significantly enriched for driver genes, including pathways in cancer, chronic myeloid leukaemia, pancreatic cancer, prostate cancer, melanoma, colorectal cancer, endometrial cancer, the $H I F-1$ signalling pathway, the $P I 3 K$ - $A k t$ signalling pathway, the $m T O R$ signalling pathway, apoptosis, and cell cycle $(\mathrm{FDR}<0.05$, data not shown).

Coexpression network analyses in PRAD. To characterise the coexpression networks of the 333 identified driver genes, WGCNA coexpression networks were constructed based on the expression correlation between driver genes in the 497 PRAD samples. As shown in Fig. 2, WGCNA analysis identified six distinct gene coexpression modules in PRAD. These coexpression modules are shown in different colours and are arranged from large to small by the number of genes they included, with 166, 58, 42, 25, 21 and 15 in the grey, turquoise, blue, brown, yellow and green modules, respectively (Fig. 2). The module-trait association analysis indicated that the yellow module was significantly positively correlated with the number of positive lymph nodes ( $\mathrm{P}<0.05$, data not shown). ITGAL, TAGAP, SIGLEC10, RAC2 and ITGA4 were the top five hub genes in the yellow module.

$C N V$ analyses in PRAD. Focal CNVs were obtained for 492 PRAD samples from the Broad Institute. Significant focal gains and deletions $(\mathrm{Q}<0.25)$ were found at 63 loci (28 amplifications and 35 deletions) in $90.45 \%$ (445/492) of the PRAD samples. Among them, deletions at 8p21.3, 13q14.13, $16 q 24.1,16 q 22.3$ and $6 q 14.3$ were the most frequent CNVs in PRAD, with occurrence rates of $59.15 \%$ (291/492), $45.53 \%$
(224/492), $42.68 \%$ (210/492), 38.21\% (188/492) and $35.98 \%$ (177/492), respectively (data not shown). PPP3CC, XPO7, RB1, BANP, ZFHX3, FREM2, RFXAP, TP53, MYCBP2 and $P T E N$ were the 10 most frequently deleted driver genes in PRAD, whereas KLF10, FZD6, MTERFD1, PTDSS1, RIMS2, $C C N E 2, C S M D 3, A D C Y 8, C Y P 11 B 2$ and GSDMD were the 10 most frequently amplified driver genes in PRAD (Fig. 3). Hierarchical clustering analysis of these 20 genes revealed three subgroups of patients with PRAD: Those with minimal CNVs (cluster 1), those with intermediate CNVs (cluster 2) and those with substantial CNVs (cluster 3), as shown in Fig. 3. Patients in the substantially altered cluster 3 exhibited significantly poorer survival rates compared with patients in the minimally altered cluster $1(\mathrm{P}=0.02, \log$-rank test, Fig. $4 \mathrm{~A})$. In addition, the tumours in cluster 3 exhibited significantly higher numbers of positive lymph nodes, higher Gleason scores and more advanced cancer stages than those in clusters 2 or 3 $(\mathrm{P}<0.05$ for all cases, Wilcoxon sum rank test, Fig. 4B-D).

Clinical features analyses in PRAD. RNA-seq and clinical features data were acquired from the TCGA database to evaluate associations between the mRNA expression of driver genes and the clinical features of patients with PRAD. Overall, 18 driver genes were significantly correlated with patient outcome at the cut-off of $\mathrm{P}<0.05$; and TP53 was frequently deleted in PRAD and significantly associated with patient prognosis (data not shown). There were a large number of driver genes negatively correlated with the number of positive lymph nodes (20 genes, data not shown), Gleason score (51 genes, data not shown), and pathologic stage (36 genes, data not shown). Similarly, several genes were positively correlated with the number of positive lymph nodes (38 genes, data not shown), Gleason score (101 genes, data not shown), and pathologic stage (78 genes, data not shown). A total of 48 genes were significantly associated with the number of positive lymph nodes, Gleason scores, and pathologic stage, including NOX4, SPOP, BRCA1, BRCA2, SMAD4 and TMED10 (Fig. 5A-D, Table I), suggesting that they may represent potential druggable targets for patients with PRAD.

Validation of randomised selection of patients with PRAD. The datasets used for somatic mutation, coexpression network, clinical feature and CNV analyses were distinct patient cohorts. To evaluate heterogeneity across the different cohorts, clinical characteristics were compared among the three cohorts of patients with PRAD. There were no significant differences in patient age, number of positive lymph nodes, Gleason scores $(\mathrm{P}>0.05$ for all cases, Wilcoxon sum rank test, Table II), patient cancer stage ( $\mathrm{P}=1$, Pearson's $\chi^{2}$ test, Table II) or survival status ( $\mathrm{P}>0.05$ for all cases, log-rank test, Table II and data not shown). These results suggested that the cohorts of patients with PRAD were randomly selected and did not significantly affect the findings of the present study.

\section{Discussion}

Cancer is a disease caused by the acquisition of somatic driver mutations that confer a growth advantage to cells (25). Genes carrying driver mutations serve a pivotal role in the formation and progression of cancer and have become a focus of 
Cluster dendrogram
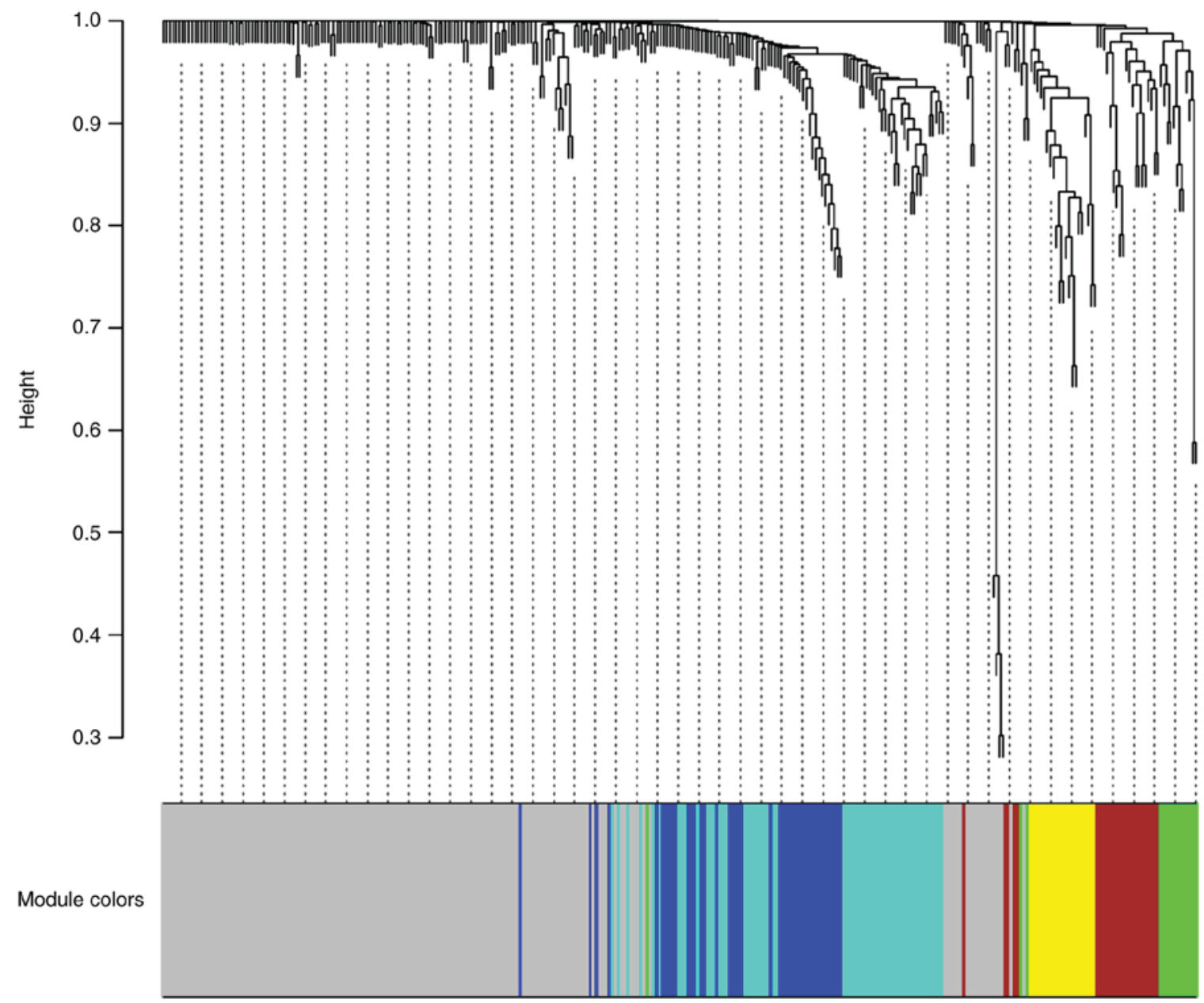

Figure 2. Clustering dendrograms of genes, with dissimilarity based on topological overlap, together with assigned module colours. Six coexpression modules were constructed and are shown in different colours.
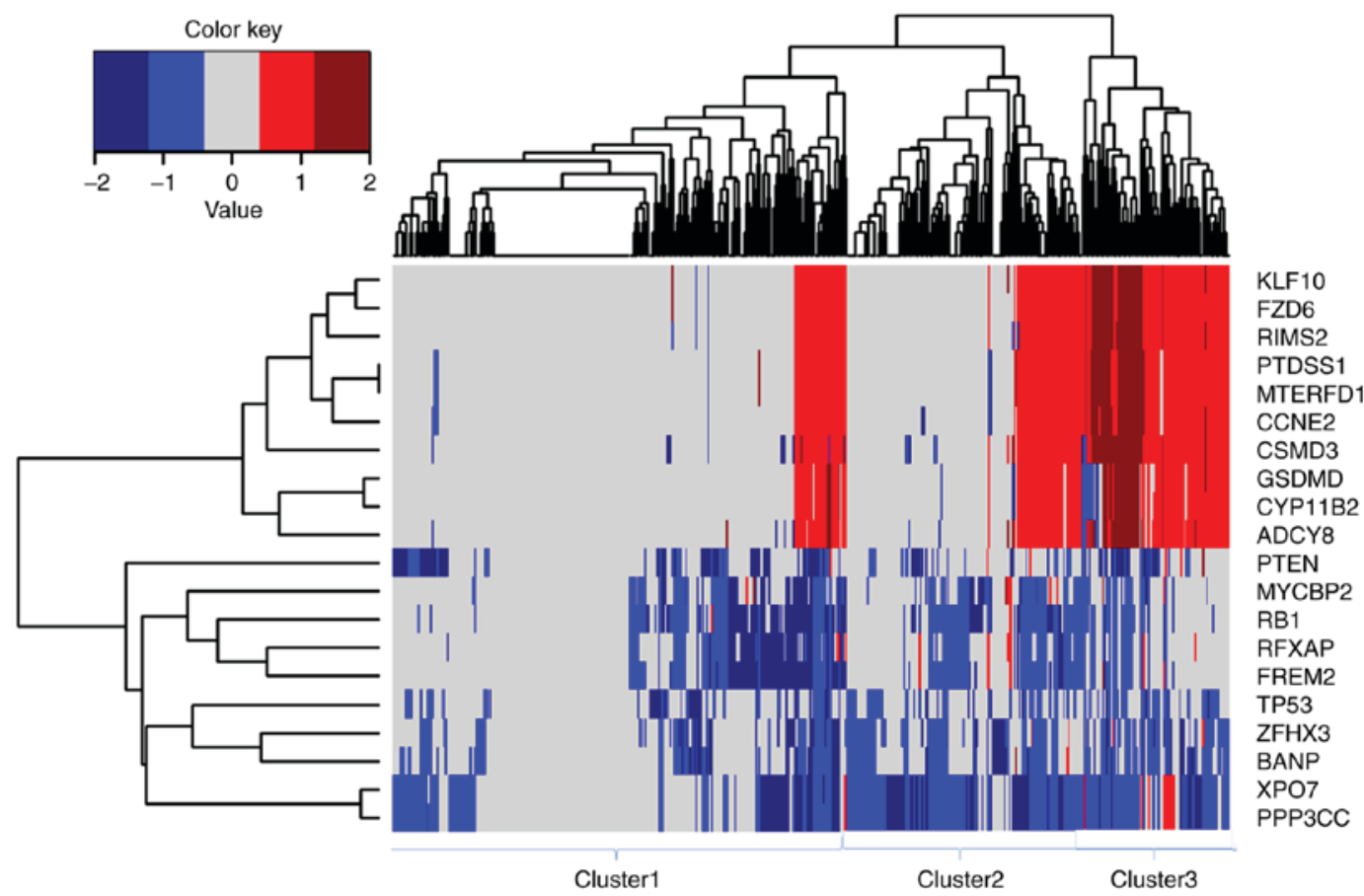

Figure 3. Unsupervised hierarchical clustering of 20 driver genes with the most frequent copy-number alterations indicates three subgroups of prostate cancer. Dark red, red, white, blue and dark blue represent high level amplification, amplification, copy-neutral, deletion and high level deletion, respectively. 
Table I. Associations between clinical features and expression of NOX4, SPOP, BRCA1, BRCA2, SMAD4 and TMED10.

\begin{tabular}{|c|c|c|c|c|c|c|c|c|c|}
\hline \multirow[b]{2}{*}{ Gene } & \multicolumn{3}{|c|}{ Number of lymph nodes } & \multicolumn{3}{|c|}{ Gleason score } & \multicolumn{3}{|c|}{ Cancer stage } \\
\hline & $\begin{array}{l}\text { Correlation } \\
\text { coefficient }\end{array}$ & $\mathrm{P}$-value & Q-value & $\begin{array}{l}\text { Correlation } \\
\text { coefficient }\end{array}$ & P-value & Q-value & $\begin{array}{l}\text { Correlation } \\
\text { coefficient }\end{array}$ & P-value & Q-value \\
\hline NOX4 & 0.21 & $<0.01$ & $<0.01$ & 0.34 & $<0.01$ & $<0.01$ & 0.33 & $<0.01$ & $<0.01$ \\
\hline SPOP & -0.18 & $<0.01$ & $<0.01$ & -0.24 & $<0.01$ & $<0.01$ & -0.13 & $<0.01$ & 0.02 \\
\hline BRCAl & 0.15 & $<0.01$ & 0.02 & 0.22 & $<0.01$ & $<0.01$ & 0.15 & $<0.01$ & 0.01 \\
\hline$B R C A 2$ & 0.16 & $<0.01$ & 0.01 & 0.25 & $<0.01$ & $<0.01$ & 0.21 & $<0.01$ & $<0.01$ \\
\hline SMAD4 & -0.18 & $<0.01$ & 0.01 & -0.18 & $<0.01$ & 0.01 & -0.13 & $<0.01$ & 0.02 \\
\hline TMED10 & -0.17 & $<0.01$ & 0.01 & -0.27 & $<0.01$ & $<0.01$ & -0.19 & $<0.01$ & $<0.01$ \\
\hline
\end{tabular}
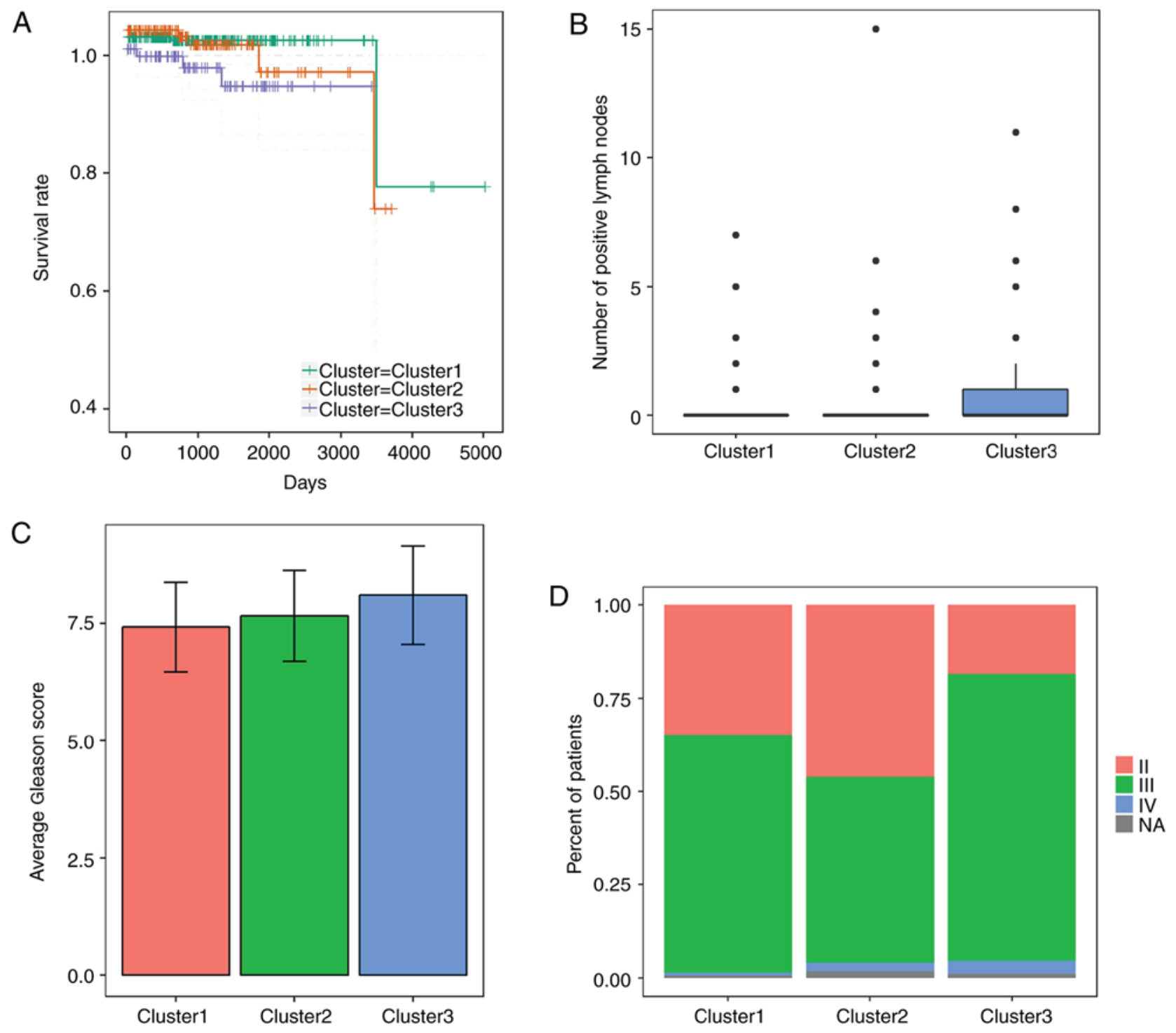

Figure 4. Differences between clusters of PRAD. Differences in (A) patient survival rates, (B) number of positive lymph nodes, (C) Gleason score and (D) cancer stage were compared among the three clusters of patients with PRAD (1-3). PRAD, prostate adenocarcinoma.

investigations in cancer genomics (4,26-29). The common approach for detecting driver genes attempts to identify genes that are significantly mutated in a cohort of cancer samples compared with the background mutation rate $(24,30)$. However, current understanding of driver genes with low mutation frequencies remains limited. In the present study, four computational tools were used to identify 333 cancer driver genes in 332 PRAD samples. In line with previously published studies, 
Table II. Comparison of clinical characteristics among three cohorts of patients with prostate adenocarcinoma for analysis of somatic mutation, WGCNA, clinical features and CNVs.

\begin{tabular}{|c|c|c|c|c|c|}
\hline Dataset & Age (years) & $\begin{array}{c}\text { Cancer stage } \\
\text { (number of patients) } \\
\text { T2A/T2B/T2C/T3A/T3B/T4 }\end{array}$ & $\begin{array}{l}\text { Number of } \\
\text { lymph nodes }\end{array}$ & $\begin{array}{l}\text { Gleason } \\
\text { score }\end{array}$ & $\begin{array}{l}\text { Survival (patients } \\
\text { alive/deceased) }\end{array}$ \\
\hline $\begin{array}{l}\text { Dataset for somatic } \\
\text { mutation analysis } \\
\text { (332 patients) }\end{array}$ & $60.63 \pm 6.88$ & 9/7/111/111/83/6 & $0.4 \pm 1.3$ & $7.58 \pm 0.98$ & $7 / 325$ \\
\hline $\begin{array}{l}\text { Dataset for WGCNA } \\
\text { and clinical feature } \\
\text { analyses ( } 497 \text { patients) }\end{array}$ & $61.04 \pm 6.81$ & $13 / 10 / 164 / 158 / 135 / 10$ & $0.45 \pm 1.37$ & $7.61 \pm 1.01$ & $10 / 487$ \\
\hline $\begin{array}{l}\text { Dataset for CNV } \\
\text { analysis (492 patients) }\end{array}$ & $60.98 \pm 6.8$ & $13 / 10 / 164 / 156 / 132 / 10$ & $0.43 \pm 1.36$ & $7.61 \pm 1.01$ & $9 / 483$ \\
\hline Statistical results & $\begin{array}{l}\mathrm{P}>0.05 \text { for } \\
\text { all cases }\end{array}$ & $\mathrm{P}=1.00$ & $\begin{array}{l}P>0.05 \text { for } \\
\text { all cases }\end{array}$ & $\begin{array}{c}\mathrm{P}>0.05 \text { for } \\
\text { all cases }\end{array}$ & $\begin{array}{c}\mathrm{P}>0.05 \text { for } \\
\text { all cases }\end{array}$ \\
\hline
\end{tabular}

WGCNA, weighted correlation network analysis; CNV, copy number variant.

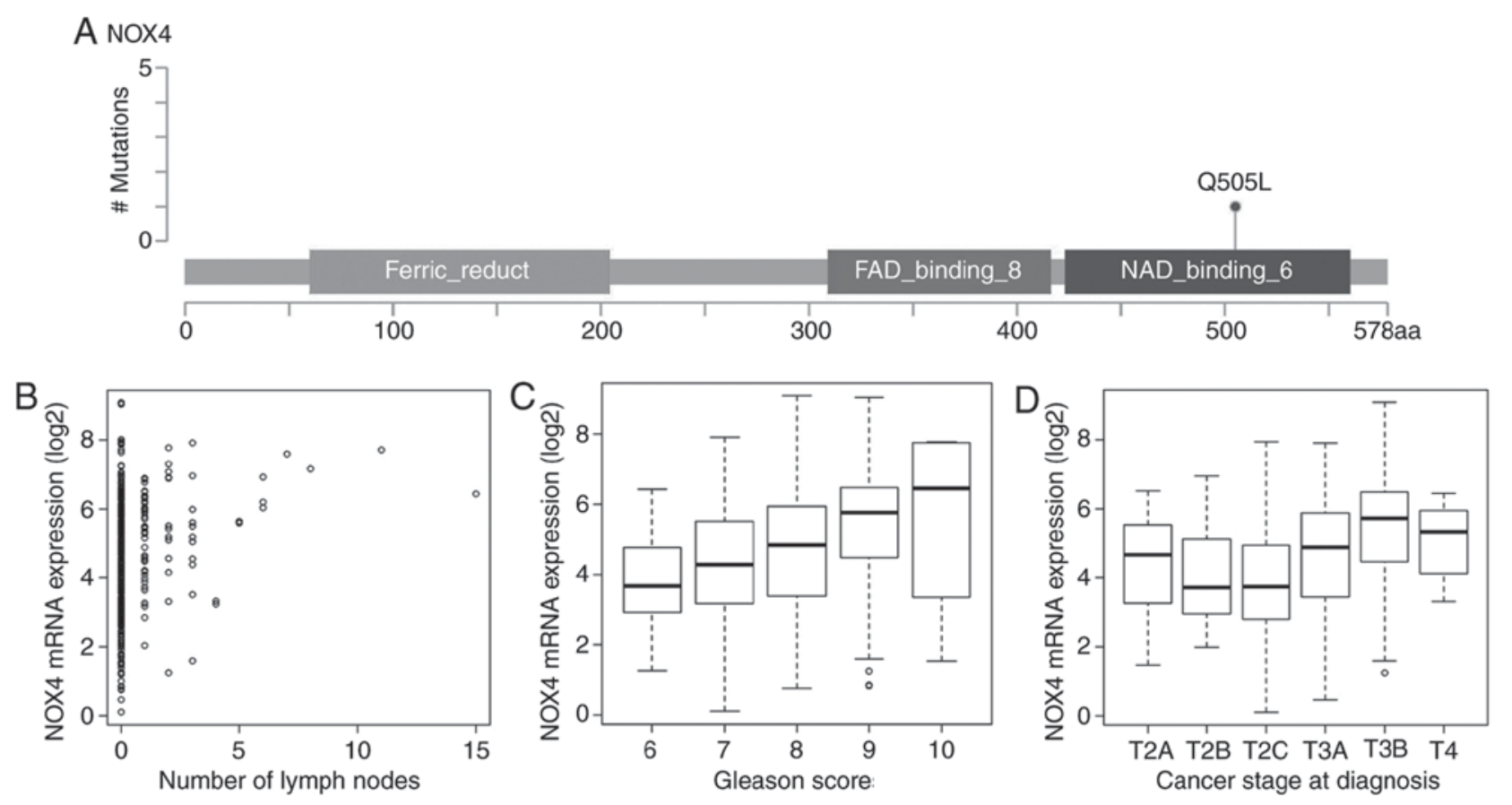

Figure 5. Clinical feature analysis of the driver gene NOX4 in PRAD. (A) A missense mutation was observed in the NAD binding 6 domain (aa 423-560), causing an amino acid change of Q505L. (B) Positive correlation between the expression of NOX4 and number of positive lymph nodes in patients with PRAD (correlation coefficient $0.21, \mathrm{P}<0.0001$ ). (C) Positive correlation between the expression of NOX4 and Gleason score in patients with PRAD (correlation coefficient 0.34, P<0.0001). (D) Positive correlation between the expression of NOX4 and pathologic stage in patients with PRAD (correlation coefficient 0.33 , $\mathrm{P}<0.0001)$. PRAD, prostate adenocarcinoma; aa, amino acid.

SPOP, TP53, FOXA1, PTEN, RB1, PIK3CA and MED12 were found to be driver genes in PRAD $(4,26)$. TP53, PTEN and RB1, which are prostate cancer tumour suppressors, were commonly altered in PRAD but primarily through copy-number loss rather than point mutations. Compared with annotated oncogene (31) and tumour suppressor gene (32) databases, the present study identified several known oncogenes, including $B R A F$, CTNNB1, PIK3CA, EGFR, HRAS and CDH1, and tumour suppressor genes, including $A T M, B R C A 1, B R C A 2, B A P 1$ and
$L R P 1 B$. The majority of driver genes exhibited low mutation frequencies, with an average mutation rate of $0.83 \%$, and were identified as driver genes in PRAD for the first time, to the best of our knowledge; these included BCOR, FRG1B, GABRA6 and $L R P I B$. Four computational tools were used to determine driver genes based on complementary principles independent of mutation recurrence, enabling the identification of recurrent and rarely mutated driver genes in a more comprehensive manner than using MutSig alone, as in a previous study (26). 
These newly identified driver genes pave the way for further experimental validation in future investigations.

In the present study, six coexpressed modules were identified, of which the yellow module was associated with the number of positive lymph nodes. ITGAL, TAGAP, SIGLEC10, $R A C 2$ and ITGA4 were the top five hub genes in the yellow module, suggesting that these genes have a large number of interactions with other genes. Therefore, these genes may act as key genes in the coexpression network. Hierarchical clustering analysis of 20 genes that were most frequently deleted or amplified revealed three subgroups of patients with PRAD. Cluster 3 tumours exhibited substantial CNVs in 20 driver genes and were associated with increased numbers of positive lymph nodes, Gleason scores and cancer stages, and a poor prognosis. Therefore, CNV analysis of these 20 genes may be of clinical value in the near future. Cytologic or surgical specimens of PRAD exhibiting high levels of CNVs in these 20 genes are expected to be associated with a poor prognosis. Therefore, more aggressive treatment or frequent follow-up may be recommended for these patients.

Finally, 48 genes were significantly associated with the number of positive lymph nodes, Gleason scores and pathologic stage, including NOX4, SPOP, BRCA1, BRCA2, SMAD4 and TMED10. The NOX4 gene encodes a member of the NOX family of enzymes that functions as the catalytic subunit in the NADPH oxidase complex. The encoded protein localises to nonphagocytic cells, where it acts as an oxygen sensor and catalyses the reduction of molecular oxygen to various reactive oxygen species (33). NOX4 gene deletions are frequent in patients with hepatocellular carcinoma, correlating with higher tumour grade. The loss of NOX4 increases actomyosin levels and favours an epithelial to amoeboid transition, contributing to tumour aggressiveness (34). The increased expression of NOX4 enhances cancer growth, progression and metastasis in HeLa cells (35), renal cell carcinoma (36) and glioma (37). The expression of NOX4 is upregulated in prostate cancer (38), and the NOX4 inhibitor diphenyliodonium inhibits reactive oxygen species generation, which decreases cell proliferation and cell migration and induces G2-M cell cycle arrest in prostate cancer cells (39). The results obtained in the present study, in combination with previously published reports, indicate that NOX4 exerts oncogenic functions in cancer.

\section{Acknowledgements}

Not applicable.

\section{Funding}

No funding was received.

\section{Availability of data and materials}

The datasets generated and/or analysed in the present study are available from the corresponding author on reasonable request.

\section{Authors' contributions}

RJ designed and guided the study. GL, YC and HY downloaded multiple omics data of somatic mutations, RNA-seq,
CNVs and patient clinical features from TCGA. XZ and YL predicted driver genes and pathways using four computational tools, and conducted WGCNA co-expression, CNV and clinical features analyses. LX and HL conducted the validation of randomized selection of PRAD patients and provided statistical advice. XZ, LX and HL wrote the manuscript. All authors read and approved the final manuscript.

\section{Ethics approval and consent to participate}

Not applicable.

\section{Patient consent for publication}

Not applicable.

\section{Competing interests}

The authors declare that they have no competing interests.

\section{References}

1. Jemal A, Bray F, Center MM, Ferlay J, Ward E and Forman D: Global Cancer Statistics. CA Cancer J Clin 61: 69-90, 2011.

2. Grignon DJ: Unusual subtypes of prostate cancer. Mod Pathol 17: 316-327, 2004

3. Shen MM and Abate-Shen C: Molecular genetics of prostate cancer: New prospects for old challenges. Genes Dev 24: 1967-2000, 2010.

4. Barbieri CE, Baca SC, Lawrence MS, Demichelis F, Blattner M, Theurillat JP, White TA, Stojanov P, Van Allen E, Stransky N, et al: Exome sequencing identifies recurrent SPOP, FOXA1 and MED12 mutations in prostate cancer. Nat Genet 44: 685-689, 2012.

5. Taylor BS, Schultz N, Hieronymus H, Gopalan A, Xiao Y, Carver BS, Arora VK, Kaushik P, Cerami E, Reva B, et al: integrative genomic profiling of human prostate cancer. Cancer Cell 18: 11-22, 2010.

6. Cancer Genome Atlas Research Network: The molecular taxonomy of primary prostate cancer. Cell 163: 1011-1025, 2015.

7. Wood LD, Parsons DW, Jones S, Lin J, Sjöblom T, Leary RJ, Shen D, Boca SM, Barber T, Ptak J, et al: The genomic landscapes of human breast and colorectal cancers. Science 318: 1108-1113, 2007.

8. Chen Y, Cunningham F, Rios D, McLaren WM, Smith J, Pritchard B,Spudich GM,BrentS, KuleshaE, Marin-GarciaP, et al: Ensembl variation resources. BMC Genomics 11: 293, 2010.

9. Tamborero D, Gonzalez-perez A and Lopez-bigas N: Genome analysis OncodriveCLUST : Exploiting the positional clustering of somatic mutations to identify cancer genes. Bioinformatics 29: 2238-2244, 2013.

10. Gonzalez-Perez A and Lopez-Bigas N: Functional impact bias reveals cancer drivers. Nucleic Acids Res 40: e169, 2012.

11. Sim NL, Kumar P, Hu J, Henikoff S, Schneider G and Ng PC: SIFT web server: Predicting effects of amino acid substitutions on proteins. Nucleic Acids Res 40 (Web Server Issue): W452-W457, 2012.

12. Adzhubei IA, Schmidt, Peshkin L, Ramensky VE, Gerasimova A, Bork P, Kondrashov AS and Sunyaev SR: A method and server for predicting damaging missense mutations. Nat Methods 7: 248-249, 2010.

13. Reva B, Antipin Y and Sander C: Predicting the functional impact of protein mutations: Application to cancer genomics. Nucleic Acids Res 39: e118, 2011.

14. González-Pérez A and López-Bigas N: Improving the assessment of the outcome of nonsynonymous SNVs with a consensus deleteriousness score, Condel. Am J Hum Genet 88: 440-449, 2011.

15. Dong C, Guo Y, Yang H, He Z, Liu X and Wang K: iCAGES: integrated cancer genome score for comprehensively prioritizing driver genes in personal cancer genomes. Genome Med 8: 135, 2016. 
16. Hua X, Xu H, Yang Y, Zhu J, Liu P and Lu Y: DrGaP: A powerful tool for identifying driver genes and pathways in cancer sequencing studies. Am J Hum Genet 93: 439-451, 2013.

17. The Gene Ontology Consortium; Ashburner M, Ball CA, Blake JA, Botstein D, Butler H, Cherry JM, Davis AP, Dolinski K, Dwight SS, et al: Tool for the unification of biology. Nat Genet 25: 25-29, 2000 .

18. Szklarczyk D, Morris JH, Cook H, Kuhn M, Wyder S, Simonovic M, Santos A, Doncheva NT, RothA, BorkP, et al: The STRING database in 2017: Quality-controlled protein-protein association networks, made broadly accessible. Nucleic Acids Res 45 (Database Issue): D362-D368, 2017.

19. Langfelder $P$ and Horvath S: WGCNA: An R package for weighted correlation network analysis. BMC Bioinformatics 9: $559,2008$.

20. Mermel CH, Schumacher SE, Hill B, Meyerson ML, Beroukhim R and Getz G: GISTIC2.0 facilitates sensitive and confident localization of the targets of focal somatic copy-number alteration in human cancers. Genome Biol 12: R41, 2011.

21. Warnes G, Bolker B, Bonebakker L, Gentleman R, Huber W, Liaw A, Lumley T, Mächler M, Magnusson A and Möller S: gplots: Various R programming tools for plotting data, 2005.

22. Therneau TM: Survival analysis. https://cran.r-project.org/web/ packages/survival/survival.pdf. Accessed November 27, 2018.

23. Andersen PK and Gill RD: Cox's regression model for counting processes: A large sample study. Ann Stat 10: 1100-1120, 1982.

24. Lawrence MS, Stojanov P, Polak P, Kryukov GV, Cibulskis K, Sivachenko A, Carter SL, Stewart C, Mermel CH, Roberts SA, et al: Mutational heterogeneity in cancer and the search for new cancer-associated genes. Nature 499: 214-218, 2013.

25. Greenman C, Stephens P, Smith R, Dalgliesh GL, Hunter C, Bignell G, Davies H, Teague J, Butler A, Stevens C, et al: Patterns of somatic mutation in human cancer genomes. Nature 446: $153-158,2007$

26. Grasso CS, Wu YM, Robinson DR, Cao X, Dhanasekaran SM, Khan AP, Quist MJ, Jing X, Lonigro RJ, Brenner JC, et al: The mutational landscape of lethal castration-resistant prostate cancer. Nature 487: 239-243, 2012

27. Cancer Genome Atlas Research Network: Comprehensive molecular profiling of lung adenocarcinoma. Nature 511: 543-550, 2014.

28. Sato Y, Yoshizato T, Shiraishi Y, Maekawa S, Okuno Y, Kamura T, Shimamura T, Sato-Otsubo A, Nagae G, Suzuki H, et al: Integrated molecular analysis of clear-cell renal cell carcinoma. Nat Genet 45: 860-867, 2013.

29. Cancer Genome Atlas Research Network: Integrated genomic characterization of papillary thyroid carcinoma. Cell 159 : 676-690, 2014
30. Dees ND, Zhang Q, Kandoth C, Wendl MC, Schierding W, Koboldt DC, Mooney TB, Callaway MB, Dooling D, Mardis ER, et al: MuSiC: Identifying mutational significance in cancer genomes. Genome Res 22: 1589-1598, 2012.

31. Liu Y, Sun J and Zhao M: ONGene: A literature-based database for human oncogenes. J Genet Genomics 44: 119-121, 2017.

32. Zhao M, Sun J and Zhao Z: TSGene: A web resource for tumor suppressor genes. Nucleic Acids Res 41 (Database Issue): D970-D976, 2013.

33. Nisimoto Y, Diebold BA, Constentino-Gomes D and Lambeth JD: Nox4: A hydrogen peroxide-generating oxygen sensor. Biochemistry 53: 5111-5120, 2014

34. Crosas-Molist E, Bertran E, Rodriguez-Hernandez I, Herraiz C, Cantelli G, Fabra À, Sanz-Moreno V and Fabregat I: The NADPH oxidase NOX4 represses epithelial to amoeboid transition and efficient tumour dissemination. Oncogene 36: 3002-3014, 2017.

35. Jafari N, Kim H, Park R, Li L, Jang M, Morris AJ, Park J and Huang C: CRISPR-Cas9 mediated NOX4 knockout inhibits cell proliferation and invasion in HeLa cells. PLoS One 12: e0170327, 2017.

36. Gregg JL, Turner RM II, Chang G, Joshi D, Zhan Y, Chen L and Maranchie JK: NADPH oxidase NOX4 supports renal tumorigenesis by promoting the expression and nuclear accumulation of HIF2 $\alpha$. Cancer Res 74: 3501-3511, 2014.

37. Shono T, Yokoyama N, Uesaka T, Kuroda J, Takeya R, Yamasaki T, Amano T, Mizoguchi M, Suzuki SO, Niiro H, et al: Enhanced expression of NADPH oxidase Nox4 in human gliomas and its roles in cell proliferation and survival. Int J Cancer 123: 787-792, 2008.

38. Meitzler JL, Makhlouf HR, Antony S, Wu Y, Butcher D, Jiang G, Juhasz A, Lu J, Dahan, Jansen-Dürr P, et al: Decoding NADPH oxidase 4 expression in human tumors. Redox Biol 13: 182-195, 2017.

39. Kumar B, Koul S, Khandrika L, Meacham RB and Koul HK: Oxidative stress is inherent in prostate cancer cells and is required for aggressive phenotype. Cancer Res 68: 1777-1785, 2008.

This work is licensed under a Creative Commons Attribution-NonCommercial-NoDerivatives 4.0 International (CC BY-NC-ND 4.0) License. 